\title{
Cerrando la Brecha de la Riqueza: Una Tercera Vía Justa
}

\section{Una Perspectiva Diferente de la Globalización \\ Económica}

"La tormenta econǿmica que ha estado azotando las economias en lod'o el globo está in tensificando una de las peores -y más desconcertantes- crisis monetarias desde que el sistema de tasas de cambio fijas se denrumbó hace un cuarto de siglo. "Asi es come The Wall Street Journal caracterizó la última serie de catásIrofes económicas mundiales'. El problema es que nadie parece tener una solución viable. Como el arliculo afirma, "Lo que hace que las crisis sean preocupantes es que no hay una solución concreta a la vista -ningún apagafuegos financiero que los gobiernos o las instituciones financieras internacionales puedan estructurar para hacer más lenta su generalización.

Este pesimismo general es intensificado por una creciente concientización de una mayorque la de cualquier Estado en el mundo, incluso la Unión Europea, que es la fuerza de la globalización económica. Esıa es la conclusión del conocido autor William Greider en su libro de 1997. Un Mundo, Listo o No: La Maníaca Lógica del Capitalismo Global (One world. Ready or Not: The Manic Logic of Global Capitalism)². Greider señala que la globalización económica dirigida por una elite financiera con el poder para mover billones de dólares casi instantáneamente de un país o otro-es una realidad que no desaparecerá. La habilidad de quienes controlan el dinero y las finanzas para derrocar a
Jefes de Estado considerados invulnerables, se evidenció con el derrocamiento del Presidente Sukarno de Indonesia.

La subordinación de los líderes políticos mundiales a quienes controlan el dinero fue predecible desde hace por lo menos un siglo cuando uno de los primeros capitalistas financieros, Mayer Anshel Rothschild, ha sido citado por decir: "Déjenme emitir y controlar el dinero de la nación, y no me importa quien escriba las leyes"

Pero para la mayor parte de gente la globalización económica significa una creciente brecha entre ricos y pobres, un ale jamiento tecnológico de los medios de producción para los trabajadores y el fenómeno de "wage arbitrage", en el cual las empresas transnacionales y las alianzas estratégicas pueden obligar a los trabajadores en mercados con altos cosios salariales a competir con maquinaria que ahorra mano de obra y con trabajadores exiranjeros cuyo costo es menor para su contratación. Esta observación, planteada por primera vez por Louis Kelso al final de los cincuentas, ha sido reforzada por muchos escritos del Centro para la Justicia Económica y Social ${ }^{1}$, en Un Mundo Listo o No cuyo autor es Greider y en dos libros de Jeff Gates en los que se han venido popularizando la revolución de los propietarios por medio del crédito de Kelso;.

Aún los Estados Unidos, que en la actualidad parece estar disfrutando en una relativa prosperidad económica en medio de un deslizamiento financiero mundial ala dcpre- sión, muestra síntomas similares. Los Estados Unidostiene una de las brechas más grandes entre "los que tienen" y "los que no tienen". Las empresas estadounidenses tienen la brecha de sueldos más amplia entre los CEO y los trabajadores de la empresa en general y está aumentando más. El bajo desempleo oculta un desplazamiento de los trabajadores por la tecnología y la mano de obra extranjera más barata. de lo que resulta una mayor incertidumbre económica e ingresos inestables por ju bilación. Para cubrir los gastos familiares, las madres se ven obligadas a entrar a la fuerza laboral, agregando presiones adicionales al bienestar de las familias.

\section{Esta falta de dirección se re-} fleja en una creciente demanda de que "algo debe hacerse". pero hay una notoria falta de algo concreto, excepto las inoperantes soluciones del pasado.

\section{¿Existe una "Tercera Vía"?}

En contraste con la confusión intelectual prevaleciente en los altos niveles de las discusiones sobre las políticas, en éste trabajo se afirma que ninguna Tercera via es una verdadera "Tercera Via" si:

No fortalece económicamente a todos los ciudadanos.

Mantiene el poder económico y social, especialmente sobre las tecnologias avanzadas. concentrados en manos de una élite. 
Mantiene a la mayor parte de la gente en una condición de dependencia servil del Estado o de otra gente.

Carece de una teoría colicrente y de principios cle justicia económica para orientar a quiencs formulan las politicas.

No tiene un sistema estructurado para cerrar la brecha entre los ricos y pobres dentro del mercado global emergente.

Sujeta la seguridad del ingreso a esquemas vulnerables de redistribución de ingresos, en vez de estimular un sistema sustentado por activos que relacionen los ingresos futuros para el consumo con la producción futura de riqueza.

Ignora el papel central cle "instrumentos sociales" tales como el dinero, el financiamiento de capital $y$ la banca central en la determinación que si el acceso al futuro crecimiento de los bienes de capital y el futuro poder económico continuará monopolizado por la elite de los ricos o si será dif undida ampliamente entre los individuos y familias.
Lógicamente, una "Tercera Via Justa" debería ser un sistema de libre mercado que fortalece economicamente a todas las personas y familias por medio de la propiedad directa y ef ectiva de los medios de producción - el último control en cualquier sociedad contra la corrupción potencial $y^{\prime}$ el abuso cle poder

Un error en que incurren muchos catedráticos $y$ economistas actualmente es equiparar la democracia y la economia cle mercado con el modelo capitalista de Wall Street, que es un sistema vertical con una creciente brecha cle la riqueza entre los ricos y los pobres. Aún cuando los gobiernos son electos clemocráticamente, no debe sorprendernos el hecho de que hay una excesiva co. rrupción en el capilalismo y el socialismo. Lord Acton nos previno hace años sobre la corruptibilidad en los sistemas donde hay concentración del poder.

Teóricos del capitalismo como Milton Fricdman no prestan atención a la concentración cic la propiedad de la tecnologia que desplaza al trabajador. Los tcóricos mirxistas lo hacen, pero concluyen que el Estado debería tener la propiedad y regular los medios de producción. Los Ǩ̀nesianos of recen una débil sintesis entre estos dos modelos de desarrollo basada en la premisa de que la mala distribución cle la propiedad es aceptable. La llamada "Tercera Via" de Clinton y' Blair sigue el modelo Kiynesiano.

En 1958, Lunis O. Kelso fue co-autor de un librok con cl reconocido académico aristotélico Mortimer I. Adler. que se centraba en una teoría seria de la justicia cconómica que representaha una cliril visión del impacto de la tecnologia en el traba jo humano y cómo las corporaciones financicras modernas han influiclo en la calidad del trabajo y de este modo en la políica y la moral de la sociedad

La mayor parte de académicos nunca pasaron del título del primer libro de Kelso y Adler, que desafortunadamente fuc titulado El Manifiesıo Capitalista. Kelso. sin embargo ha ganadof ama internacional como el inventor del Plan de Propiedad para los Empleados de las Acciones de la Compañia (Employee

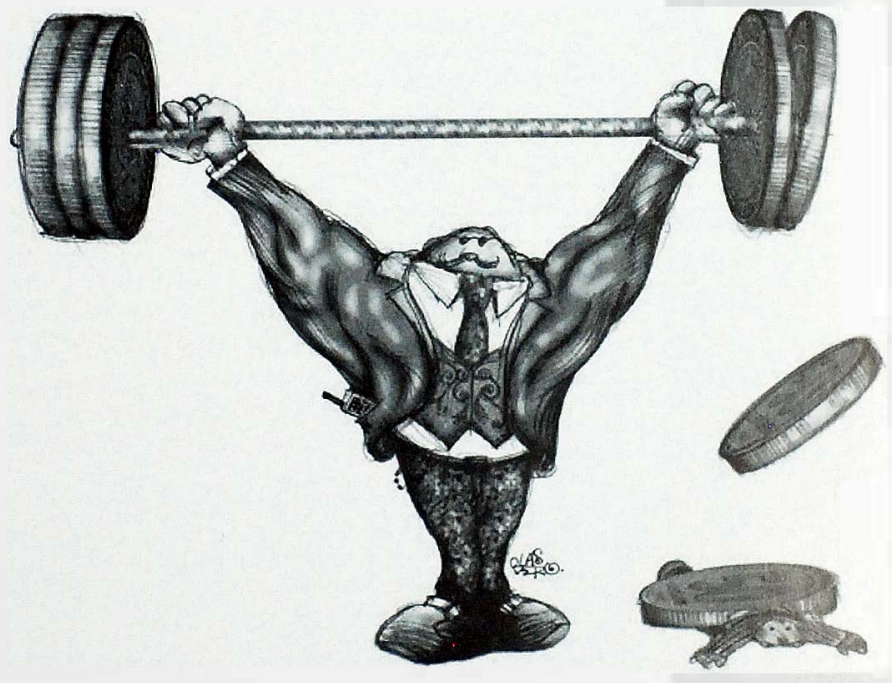




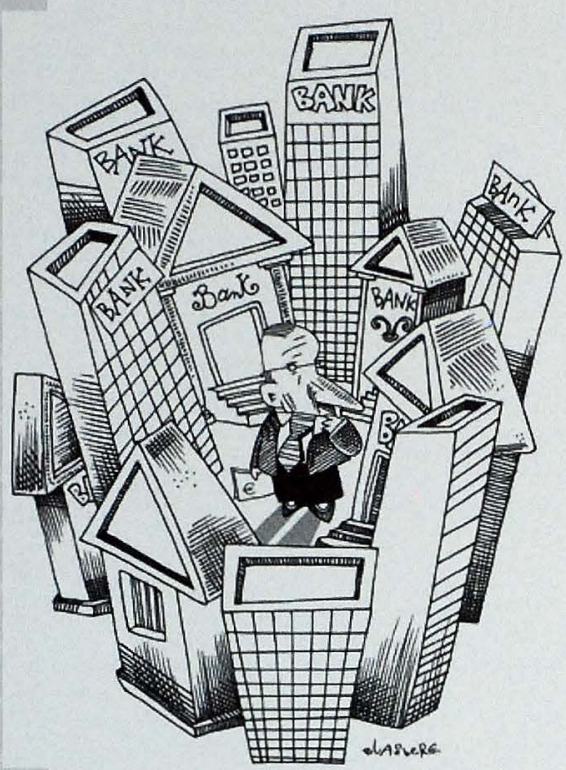

Stock Ownership Plan o ESOP), que es uno de los instrumentos que él desarrolló para democratizar el acceso al dinero y al crédito. Sorprendentemente, sinembargo, su penetrante visión y su teoría general han sido trivializados y prácticamente ignorados en el ambiente academico y en la corriente prevaleciente de los medios de comunicación. Un destacado libro de 1999 por Robert Ashford y Rodney Shakespeare sobre el nuevo paradigma económico concebido por Kelso explica porqué los economistas Iradicionales tienen dificultad para entender y resolver los problemas que surgen de la globalización económica?

El análisis revolucionario de Kelso le permitió resolver el enigma económico de cómo la Ley de Say sobre los mercados - rechazada tanlo por Marx como por Keynes- puede lograr un crecimiento sostenible y balanceado en la economía global moderna. Concentrándose en los medios porloscuales la gente común podría convertirse en propietaria de los bienes de capital productivos y tener una mayor participación en el proceso económico, Kelso proporcionó al sistema los mecanismos teóricos y práclicos, como el ESOP, para implementar en el mundo una más amplia y generalizada propiedad.

\section{Lecciones de la Primera Revolución Estadounidense.}

La relación entre una amplia distribución de la propiedad y la democracia política fue evidente para los fundadores de los Estados Unidos de América. Esta creencia se relleja en la Declaración de Derechos de Virginia de 1776, que precedió a la Declaración de Independencia y la Declaración de los Derechos. A partir de los tres derechos fundamentales e inalienables identificados por Jolın
Locke, la Declaración de Derechos de Virginia estableció que asegurar "la Viday la Libertad, con los medios para adquirir y poseer la propiedad", es el más alto objetivo por el cual es creado todo gobierno justo.

El poder existe en la sociedad, independiente de que existan o no los propietarios. Sin embargo, si aceptamos el señalamiento de Lord Acton de que "el poder tiende a corromper y el poder absoluto corrompe siempre", nuestra mejor protección contra la corruptibilidad de la concentración del poder es la descentralización del poder, especialmente el poder económico. Si Daniel Webster también está en lo correcto cuando dice: "el poder -natural y necesariamente- nace de la propiedad", entonces la democratización de la propiedad es esencial para la democratización del poder.

En el mundo económico la propiedad desempeña la misma fun- 


\section{Cerrando la brecha de la riqueza: una tercera vía justa}

ción de dilusion del poder yue el volo tience en la politica. Pero hate mis. Convierte alvotante, ecomomicimente independiente de yuienes déntan el poller.

\section{Viendo más allá del Socialismo y el Capitalismo}

Tante el socialismo como el capitalismoconcentran el poder economico en los niveles superiores. No es una diferencia importante yue en cl capitalismo la concentración se clá en manos privadas $y$ en el socialismo, en el Estado. Ambos sistemas son excesivamente maltcrialisias en sus principios básicos y en su visión gencral. Ambos, en diferentes formas. degradan al trabajador. Ambos sistemas concrelan escyuemas económicos que ignoran e inhiben cl clesarrollo intelectual y espiritual.

Las mezclas de los dos sistemas. que en Estados Unidos es la llamada "economía mixta" o los moclelos eur opeos y' japonés. se dif erencian únicamente en el grado de su injusticia social, corrupcion, ineficiencia economica, inseguriclad humana y alienación que penerra todos los niveles cle las socieclacles cli- vidiclas en clases. Entonces, iCuál seria la verdadera "Terecra Vial para avallyar hacia ma socicdad mais libre, ma is juslat y sin clases económicals?

Todos los modelos convencionales del "sistema de salarios" para el desarrollo incurren en un error fatal. La derccha se mantiene ciega a las barreras institucionales para ampliar el número de propieıarios, limitando implícitamente la propiedad cle los bienes produclivos cle capital a una elite minima. En consecuencia, resulıa que la gran mayoría cle trabajaclores perciben ingresos unicamente de la venta de su fuerza laboral, en abierta competencia con la tecnologia avanzada y una creciente fuerza laboral munclial. Lo anterior, en clelinitiva, recluce al trabajador a un insumo de la producción. Entonces puede ser comprado más barato y forzado al clesempleo si los empresarios deciclen dirigirse clonde el costo de la mano de obra es más bajo o bien sustituir a los trabajaclo. res por máquinas. Las exclusiones cle los sistemas de financiamiento convierten a los paises subdesar rollados dependientes, en forma permanente. de las inversiones cle capital extranjero para mantener funcionando sus economias. Los ciucladanos en e cemero ye nl a izcuierda vuelven los ojos al gobierno, no al sisiema de mercialo, para resolver los probiemas ignoralos por la derecha.

Historicamentc cl capitalismo y el socialismo han violado los derechos yuc lienen los propictarios de los biencs productivos sobre el producto. Texlo excedente es tomado de los propietarios y trabajadores procluctivos y redistribuido entre los que no son propiclarios y son improGluctivos. Esto deja más poder económico en manos del Estado de los (yuc es saludable para alcanzar una verdatera justicia económical y secial para todos

\section{Mas allá del Sistema de} Salarios

Toclos los sistemas cle salarios ignoran uno o más cle los llamaclos "Cuatro Pilares" de la Tercera Vía, cuatro principios esenciales e interdepenclientes para construir una economia cle mercado más justa

Propic lad más amplia de los biencs productives

Poder económico limitado dcl Estado

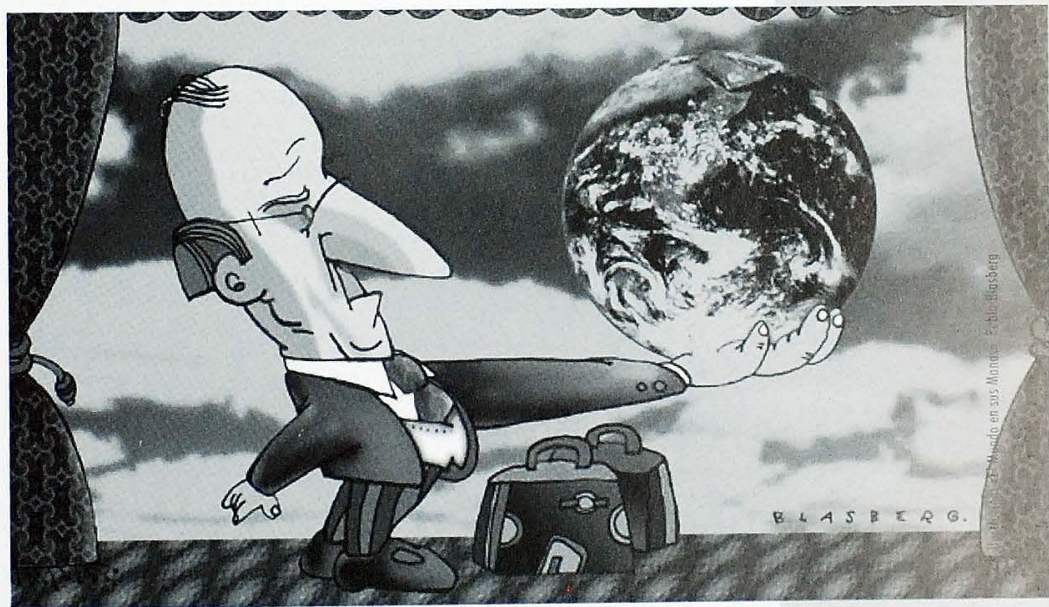


Restauración de mercados libres y abiertos

Restauración de la propiedad privada

Ampliación del sistema de propiedad: La omisión moral de todas las economías actuales-

Uno de los problemas más cruciales que Marx estudió en sus teorias económicas fue que la propiedad de los bienes productivos "El Capital"- estaba limitado a muy pocos. Como resultado, ningún sistema de mercado de alta tecnología podría producir un desarrollo sostenible, ya que el sector laboral tendria únicamente sus trabajo para vender, en competencia directa con la tecnología sustitutiva de la mano de obra y una creciente población mundial de trabajadores dispuestos a trabajar por salarios menores. Marx tenia razón: el sistema tiene las semillas de su propia destrucción. Desafortunadamente, la solución de Marx a esta incompatibilidad entre el crecimiento tecnológico de la productividad y el mercado. que descansa en los ingresos para el consumo, fue concentrar aún más el control sobre la riqueza productiva y el poder. al establecer obligatoriamente la propiedad del Estado sobre el capital productivo. De ahí resultó una enorme concentración de riqueza y poder en las manos de una nueva elite política.

El problema real que Marx encaró al confrontar el capitalismo no fue, sin embargo, la propiedad privada de la propiedad productiva, sino la concentración de la propiedad. Si cambiamos el enfoque de Marx, haciendo de cada trabajador un propietario de una creciente porción de la propiedad productora de ingresos, ello nos permitiria - por otra partealcanzar la justicia económica para todos, manteniendo al mismo tiempo un me jor crecimiento sostenible dentro de la disciplina de una economia libre basada en el mercado.

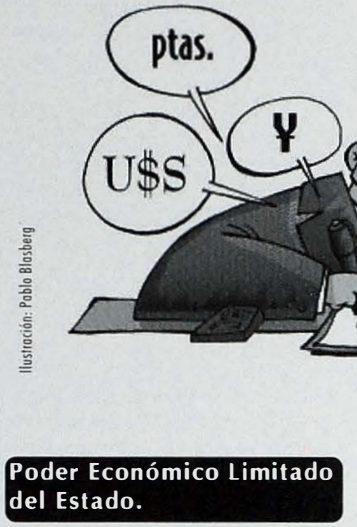

Limitar el poder económico del Estado significa esencialmente el objetivo de pasar la propiedad y el control de la producción y la distribución del ingreso del Estadoa la Sociedad. Para lograrlo, el poder económico del Estado deberá limitarse únicamente a:

Estimular un crecimiento sostenible que mejore la calidad de vida, controlando los abusos en el sector privado;

Terminar con los monopolios económicos y los privilegios especiales;

Eliminar los obstáculos para una mayor igualdad de oportunidades de ser propietario, esencialmente por medio de la reforma en el poder de creación de dinero de la banca central, para posibilitar un acceso mayor al crédito a bajo costo, como forma de ampliar la propiedad y lograr el fortalecimiento económico de los trabajadores;

Prevenir la inflación manteniendo la estabilidad monetaria para un desarrollo sostenible;

Proteger la propiedad, haciendo cumplir los contratos y resolviendo las disputas;
Promover sindicatos democráticos para negociar la propiedad y los derechos de los trabajadores;

Proteger el medio ambiente: y

Proporcionar redes de seguridad social para las emergencias.

Dentro de estos límites el Estado promovería la justicia económica para todos los ciudadanos. Coincidente con este objetivo, sería el de reducir los conflicios humanos y el desperdicio, estableciendo un ambiente institucional que estimule a aumentar la eficiencia económica y la creación de nueva riqueza para ellos y para el mercado mundial. El aumento en la producción también aumentará los ingresos totales para objetivos legítimos del sector público, reduciendo la necesidad de la distribución del ingreso por medio de impuestos sobre la renta confiscatorios y las contribuciones del seguro social.

\section{Restauración de los Mercados Libres y Abiertos.}

Los precios justos, los salarios justos y las utilidades justas son establecidos mejoren un mercado libre, abierto y democrático donde la soberanía del consumidor prevalez- 
ca definitivamente. Si suponemos una futura democratización económica en la propiedad de los medios de producción, las preferencias económicas o sea, "los votos" respecto a los precios y salarios inciden en la determinación de los valoreseconómicos en el mercado.

El establecimiento de un mercado libre y abierto se lograría eliminando gradualmente todos los privilegios especiales y los monopolios creados por el Estado, reduciendo todos los subsidios excepto para los miembros más necesitados de la sociedad, eliminando las barreras para el comercio y el trabajo libres, suprimiendo todos los métodos artificiales, no voluntarios de determinación de los precios, salarios y utilidades. Esto resultaría en una descentralización de las preferencias económicas y en el fortalecimiento de cada persona como consumidor, como trabajador y como propietario.

La distribución de la riqueza supone creación de riqueza, y los progresos tecnológicos y los sistemas avanzados -según estudios recientesson determinantes del $90 \%$ del aumento de la productividad en el mundo actual ${ }^{8}$. Asi, el crecimiento balanceado en una economía de mercado depende de la distribución de los ingresos por medio de la propiedad individual de los medios de producción. Las fuentes tecnológicas del crecimiento de la producción estaría entonces automáticamente asociados a los ingresos para el consumo basados en la propiedad, necesarios para adquirir nueva riqueza en el mercado. De este modola Ley de Say de los Mercados -que ambos, Marx y Keynes trataron de refutar-sería una realidad cierta por primera vez desde que comenzó la Revolución Industrial.

\section{Restauración de la Propiedad Privada.}

Los derechos del propietario sobre la propiedad privada son fundamentales en todo orden económico justo. La propiedad asegura las preferencias individuales y es la protección fundamental de todos los otros derechos humanos. Al destruir la propiedad privada, la justicia es negada. La propiedad privada, al contrario de los que Marx pensaba, es el vínculo individual con el proceso económico, de la misma manera que el voto secreto es el vínculo o relación con el proceso político. Cuando alguno falıa, el individuo está desconectado o "marginado" del proceso.

Dinero y Crédito para Construir una Justa Economía de Mercado

El control sobre el dinero y el crédito (por ejemplo, el capital financiero), determina en buena medida quienes serán los propietarios y los que controlarán el capital productivo en el futuro. Definitivamente. Rothschild tenia razón, como señalamos antes.

Una cuestión esencial en la discusión respecto a la Tercera Vía es si aquellos que crean el dinero y controlan el crédito actualmente. emplearán el dinero y el crédito en forma que excluya a la mayoría de la gente de su participación como propietarios y en las ganancias. $\mathrm{O}$ bien, ¿Despertará la gente para demandar la estructuración de los sistemas monetarios y crediticios actuales, para liberarse de la contínua dominación económica por los pocos que controlan la vic ja riqueza tradicio nal?

Cuando se presenta la cuestión del dinero y su creación, olvidamos algunas veces que el dinero es algo hecho por el hombre, $y$ que es moralmente neutral y si es bueno o malo, depende únicamente de cómo es creado y cómo es empleado. Igual que el voto secreto en política, el dinero es también un "bien social", una invención de la civilización moderna, un medio para medir los valores económicos y permitir a la gente participar en la economía de mercado.

Y eso es lo esencial del problema. El dinero es creado y el crédito es concedido actualmente de ma- nera que mantiene a los ricos con su riqueza y a los pobres en su lugar. El crédito de consumo, por e jemplo, es accesible prácticamente a cualquiera. mientras que el acceso al crédito (le capital (es decir "productivo") está restringido en su uso a aquellos que pueden cumplir los requisitos acostumbrados de garantía. por e jemplo, los ricos. Entonces. los pobres y la clase media pueden obtener sólo el crédito más arriesgado y más caro, mientras los ricos tienen un crédito de bajo costo y menos arriesgado. Es mis que una certeza repetida que se necesita dinero para hacer dinero o que los prestamistas únicamente conceden crédito de capital a quienes no lienen necesidad de prestar.

Concentremos nuestra atención en el trillón de dólares de los Esıa dos Unidos, del crecimiento de los activos de capital de los sectores público y privado de los Estados Unidos. que consiste en nueva tecnologia. planta y equipo, infraestructura física y espacios arrendables. Es de cir un aumento en el crecimiento de $\$ 4.000$ (dólares estadounidenses), para cada hombre, mujer y niño $y$ éstos activos productivos serán financiados en forma que no aumentan el número de nuevos propietarios. Si el financiamiento, es decir, el crédito de capital llegara a ser universalmente accesible como el voto político. los bienes de capital se convertirian en una fuente creciente de ingresos independientes de capital para todos $y$ para sus familias.

Lo que hace particularmente especial al crédito de capital, es que por su natutaleza genera más riqueza o es "autoliquidable". Es decir, que el crédito cle capital está limitado a la adquisición de activos que se espera se paguen ellos mismos con los ingresos generados por la financiación del proyecto de inversión cle capital. y en adelante se espera que ésos activos generen una continua corriente de ingresos a quien sea el propielario de esos activos. El crédito de capital es por naturaleza anti-inflacionario y entonces, cualquier pago de crédito debe provenir de otros recursos del usuario. Cuando su utili- 
zación es amplia. el crédito de consumo reduce considerablementc el poder adquisitivo del usuario.

N hacer accesible el crédito de capital sobre una base democratical. la sociedad impulsa a la generalidad hacia la atto-suticiencia e independencia económica. La amplia generalización de la riqueza y el poder. sirve como el control último para evilar el abuso de poder por parte del Estado o de las mayorías contra las minorias o cle algunos grupos de citldadanos en particular. Para ilustrar lo anterior. consideremos que si el crecimiento anualdelas necesidades de capital de los Estados Unidos fue financiado por bancos privados por medio de la capacidad de creación de dinero del Sistema de la Reserva Federal, como lo propuso Kelso. un niño que naciera ahora podria retirarse con ingresos en dividendos anuales de \$30,000 (dólares norteamericanos) y con un patrimonio de $\$ 200.000$ para de jar a su familia, sin costo alguno para los contribuyenIes $^{4}$.

\section{Una Nueva Visión del Futuro: La Transformación del Trabajo Humano}

La Tercera Via Justa es una filosofia moral y un proceso evolutivo para transf ormar el clima institucional - los sistemas legales, financieros. culturales y morales - y en consecuencia democratizar el poder económico y mejorar la calidad de vida de todos.

En su esfuerzo para "hacer de cada trabajador un propictario", la Tercera Via Justa reconoce que por naturalezal toda persona es un trabajador. En el marco conceptual de un sistema de salarios, el "trabajo" ha sido despojado de mucha cle su dignidad, limitado únicamente a aquella parte del objelivo humano ref erido a "ganarse la vida". Sin embargo, en un sentido más amplio el trabajo implica formas de la actividad humana física, mental y espiritual, desde el trabajo manual hasta la meditación.
La Tercera Vía Justa lucha por liberar a un mayor número cle Irabajadores cle aquel trabajo que puede hacerse por medio de máquinas. P'ara aquellos que continúen trabajando en la economia de men:ado. la Tercera Vía Justa transforma cmpresa por empresa, desde la práctica de un sistema de salarios lleno de conflictos hasta la cultura más anplia de una propiedad permanente. Esta nueva cultura del trabajo ha sido llamada "Administración Basada en Valores" (Value Based Management - VBM). que refleja los principios de justicia económicà de Kelso-Adler ${ }^{10}$.

Conforme al paradigma de la Tercera Via Justa. la forma más ele- vada de Irabajo no es el Irabajo económico sino el "Irabajo de descanso" no remunerado - el Irabajo de construir una civilización que ninguna máquina puede hacer. A lo largo de la historia, el trabajo creativo ha sido principalmente acometido por sujetos independientes en sus ingresos, aquellos que fueron apoyados por un patrono o por el trabajo de alguien más. La Tercera Via Justa proporciona medios por los cuales más gente puede realizar "Irabajo de descanso" como una parie importante de sus vidas y ser sostenidos por los ingresos de un capital independiente producido por sus "esclavos tecnológicos”.

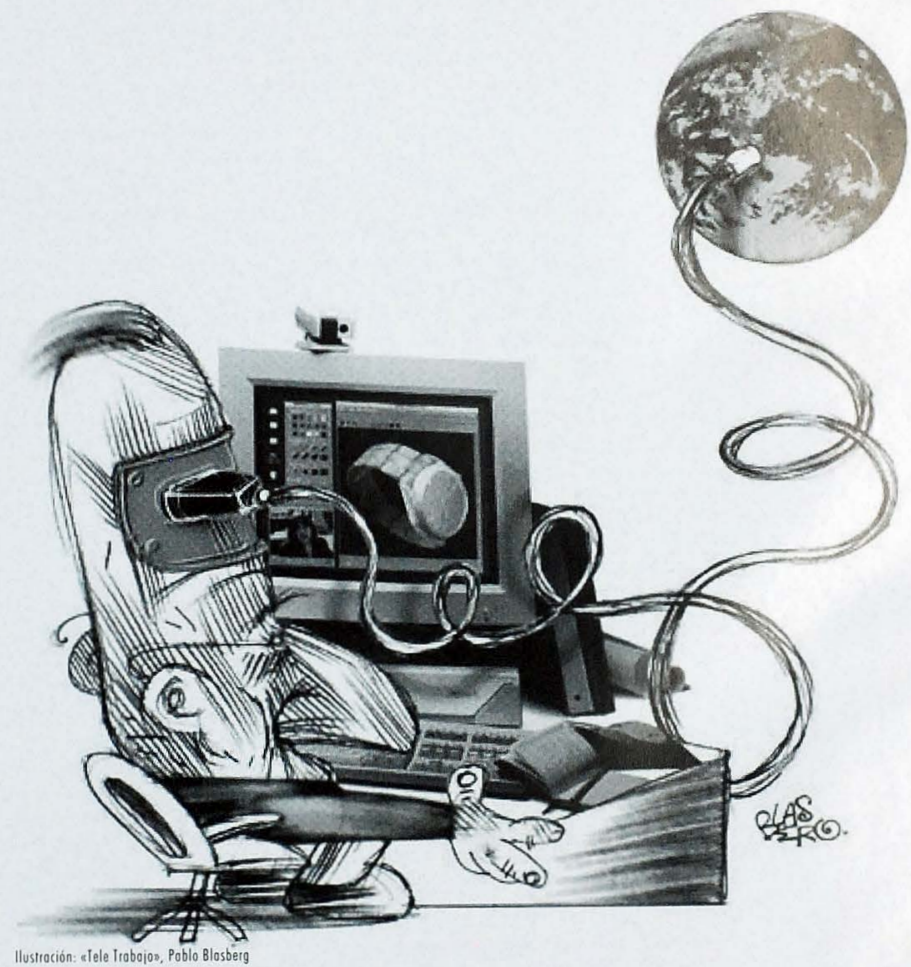


En busca de la Justicia Económica, no una Utopia

La humanidad probablemente nunca alcanzará el sistema económico "perfecto" en el cual toda tarea pesada y monótona sea eliminada y cada quien tenga la libertad de hacer el trabajo que prefiera. Sin embargo, antes de que pase la oportunidad, ha llegado a ser imperativo para todas las economias del mundo continuar creando nuevas tecnologias que mejoren los sistemas de vida para asegurar que la propiedad y su control sea sistemáticamente difundida para su extensión entre los individuos y sus familias. La alternativa es un péndulo que se mueve entre capitalismo y socialismo, en el cual cualquier período de estabilidad sólo sirve como preparación para el siguiente cambio violento.

Un enfoque fundamental en todas las reformas de la Tercer Via respecto a la banca central. la tributación y otras leyes que inciden en la economia, debe ser para la democratización del acceso al crédito productivo. Cómo se puede alcanzar esta democratización - la calendarización, las prioridades y los procedimientos - son problemas sociales que cada sociedad debe determinar por si misma.

Por muchos atios el mundo capitalista se ha cuidado del socialismo. En este extranio momento de la historia y para proteger a los ciudadanos de la pérdida de soberania económica en el modelo capitalista de IVall Street para la globalización económica, todas las naciones del mundo tienen la oportunidad de implementar una nueva e incruenta revolución económica para sus ciudadanos, que es consistente con la visión no realizada sobre la propiedad y los ideales de la civilización mundial de los grandes pensadores. En su búsqueda por una vida mejor, los ciudadanos de las economías en desarrollo y en iransformación -así como de aquellos que viven en los paises desarrollados- necesitan algo mejor que los sistemas anticuados y deshumanizantes del socialismo v capitalismo tradicional. Las naciones tienen ahora el poder cle crear nueva propiedad para los pobres, sin quitar la propiedad existente a los ricos. En otras palabras, hay adelante. otro modelo de globalización económica. una verdadera y Justa Tercera Via
Norman G. Kurland, abogado y economista, es Presidente del Centro para la Justicia Económica y Social, que es una organización sin ánimo de lucro para la investigación de la educación, que se encuentra en Arlington, Virginia. De 1965 a 1976 juntamente con Louis Kelso y su Consejo de Washington, promovieron una política nacional de ampliación de la propiedad, que generó la aprobación de más de 20 leyes aprobadas por el Congreso. En 1985 fue presidente altemo del Equipo de Estudios del Presidente Reagan en el Proyecto de Justicia Económica que recomendó políticas de reforma para impulsar la democratización económica en América Central y el Caribe. Entre sus logros está el concepto de doble nivel de la tasa de interés y otras realizaciones.

Michacl D. Grancy, es Contador Público Certilicado y Director de Investigaciones del CJES y administra ESOP para varias compafías norteamericanas propiedad de los empleados. CJES.

Dawn K. Broliawn, es Director de Comunicaciones del

Trabajo presentado a la Sección IV. IVorkshop 452 "¿.Existe una Tercer Vía?" Conferencia Seniestral de la Internacional Society for the Study of European Ideas, Universidad de Bergen. Noruega. Agosto It- IS. 2000. Traducción de Alvaro Magatta. Expresidente de la República de El Salvador.

"As Currency Crisis Spreads. Need of a Cure Grows More Pressing-" The Wall Stect Journal, 24 Agosto. 199S.

William Grcider. One World: Ready or Not: The Manic Logic of Global Capitalism (New York. NY': Simon \& Slıuster. 1997.

Fraderick Morton. The Rollıschilds. A Family Portrait (New York. NY: Atleneum. 1962).
Ver Jolin H. Miller. ed.. Curing IVorld Poverty: The New Role of Poverty (St. Louis. MO: Social Justice Review and the Center for Econonic and Social Justice. 1994).

Jeff Gites. The Ownership Solution. Reading. MA: AddisonIVeslev. 199S: and Democracy al Risk. Cambridge, MA: Perseus Publishing. 2000).

CEO son las iniciales de Chicf Exccutive Orficero sea los funcionarios del nivel miss alto de una emipresa como los presidentes. directores, gerentes. elc.

${ }^{-}$Louis O. Keisoy Mortimer J. Adler. The Capitalisı M faniliesı. (Ncw'York. NY': Random Illouse, 1958).

Robert Asliford y Rodney Sliakespeare, Binary Economies: The New Paradign (Lanham. Md.: University Press of Ánerica, 1999).

"Jołm W. Kendrick, "ProductivityTrends and Recent Slowdown Historical Perspective. Causal Factors. and Polcy Options, Contemporary Economic Problems, 1979. American Entcrprise Institutc; también R.M. Solow, en K.J. Arrow, S. Karlin y P. Suppes, eds. Mathenatical Methods in the Social Sciences, 1959. pp. 89-10.4. Stanford Uniniversity Press. 1960. También: Edward Denison. "Accounting for United States Economic Growtly: 1929-69," Washington D.C.: Brookings Institution. 1974, y Accounting for Slower Economic Growill: The United States in the 1970s, IVashington D.C.: Brookings Institution. 1979

" Ver "Saving Social Security" en http://wwwcesj.ocgilibrani' rcforms/socialsc curity/htul. el sitio web del CESJ.

10 Ver Dawn K. Broliawn, -Value-Based Management: A Framework for Equity and Justice in teli WVorkplace"; y Norman G. Kurland. "Economic Justice in the Agc of The Robot", en Curing World Poicrty: Ibid. 